This item was submitted to Loughborough's Research Repository by the author.

Items in Figshare are protected by copyright, with all rights reserved, unless otherwise indicated.

\title{
Systematic control on energy recovery of electrified turbocharged diesel engines
}

PLEASE CITE THE PUBLISHED VERSION

http://dx.doi.org/10.1109/CDC.2015.7402427

PUBLISHER

(C) IEEE

VERSION

AM (Accepted Manuscript)

LICENCE

CC BY-NC-ND 4.0

REPOSITORY RECORD

Zhao, Dezong, and Richard Stobart. 2017. "Systematic Control on Energy Recovery of Electrified Turbocharged Diesel Engines”. figshare. https://hdl.handle.net/2134/24485. 


\title{
Systematic Control on Energy Recovery of Electrified Turbocharged Diesel Engines
}

\author{
Dezong Zhao, Member, IEEE, and Richard Stobart, Member, IEEE
}

\begin{abstract}
Recovering energy from exhaust gas is seen as the promising solution to save fuel consumption of diesel engines, where the key issue in maximizing fuel economy benefits is the management of energy flows in the optimal way. This paper proposes a systematic control strategy on both energy management and air path regulation of an electrified turbocharged diesel engine (ETDE). The Energy management and air path regulation is formulated as a multi-variable online optimization problem with constraints. The equivalent consumption minimization strategy (ECMS) is employed as the supervisory level controller, to calculate the optimal energy flow distribution. An explicit model predictive controller (EMPC) is developed as the low level controller to implement the optimal energy flow distribution. The two controllers work together as cascaded modules in real-time, while simulation results based on a physical model show the superior performance over the conventional distributed single-input single-output (SISO) control method.
\end{abstract}

\section{INTRODUCTION}

Today over one quarter of the world's energy is consumed in the transportation sector, with about $23 \%$ being consumed by heavy duty vehicles [1]. Diesel engines are predominantly used in heavy duty vehicles and off-highway applications due to their high thermal efficiency. Driven by the rising fuel costs and environmental pressures, stricter legislation are proposed for diesel engines in terms of fuel efficiency and exhaust emissions. In internal combustion engines (ICE), between $22 \%-46 \%$ of fuel energy is lost to the environment through the exhaust gas [2]. Therefore, recovering energy from exhaust emissions has substantial potential in improving fuel economy and has attracted considerable development interest.

Electrified turbocharger is a critical technology in energy recovery systems, which consists of a variable geometry turbine (VGT), a compressor, and an electric machine (EM) within the turbocharger bearing housing. The use of an EM to modify turbocharger torque is referred to Electric Turbo Assist (ETA). Hybridization of the turbocharger is more efficient than feeding energy to the crankshaft, for the pumping losses are reduced. In specific engine operating regions, the fuel economy benefit of using an electrified turbocharger is up to $10 \%$ [3].

The electrified turbocharger has attracted the mainstream diesel engine manufacturers for decades [3]-[5]. In [6] and [7], the improvements to the engine transient response using an electrified turbocharger were investigated from experimental and theoretical aspects respectively. Stepping

D. Zhao and R. Stobart are with the Department of Aeronautical and Automotive Engineering, Loughborough University, Loughborough LE11 3TU, U.K. (e-mail: d.zhao2@lboro.ac.uk, r.k.stobart@lboro.ac.uk). further, [8] and [9] evaluated the potential of an electrified turbocharger in both turbo-lag reduction and fuel consumption saving. Experimental investigations into the efficiency characterization of an electrified turbocharger through experiments with a heavy duty diesel engine have been made in [10], [11]. All of the research results agree that the development of a systematic control system is essential in exploring the maximum benefits of the electrified turbocharger.

The control of a turbocharger is challenging because of strong couplings between the VGT loop and the exhaust gas recirculation (EGR) loop for the actuators are both exposed to the exhaust gas. In an electrified turbocharged diesel engine (ETDE) as shown in Fig. 1, the couplings are more complicated, due to the VGT and EM being both mounted on the same turbine shaft and therefore both affecting the exhaust pressure. The control of the ETDE is still in its infancy. This paper proposes a systematic strategy to regulate energy flows in an ETDE, with the following main contributions:

1) A two-level structure of energy management and air path regulation is established, while the controllers on the two levels work together as two cascaded functions.

2) The equivalent consumption minimization strategy (ECMS) algorithm is designed as the supervisory level controller to implement the on-line energy management.

3) An explicit model predictive controller (EMPC) is designed as the low level controller to regulate the air path dynamics, in dealing with internal nonlinearities and external constraints on actuators.

4) The proposed control strategy is successfully applied on a physical model to optimize fuel economy, while all emissions constraints are satisfied.

The paper is organized as follows. After the introduction in section I, the ETDE model is described in section II. The control problem is formulated in section III. The energy flow regulation strategy is presented in section IV. Validation results of the strategy are demonstrated in section V. Finally, the conclusions are summarized in section VI.

\section{Electrified Turbocharged Diesel Engine MODEL}

In Table I, the variables and related parameters of the ETDE illustrated in Fig. 1 are listed. A switched reluctance motor (SRM) is selected as the EM for it is an excellent option in extra-high speed applications thanks to its simple structure. The EM can work in both assist mode and generator mode. In assist mode, the EM extracts energy from the battery to improve the engine transient response, or provide 


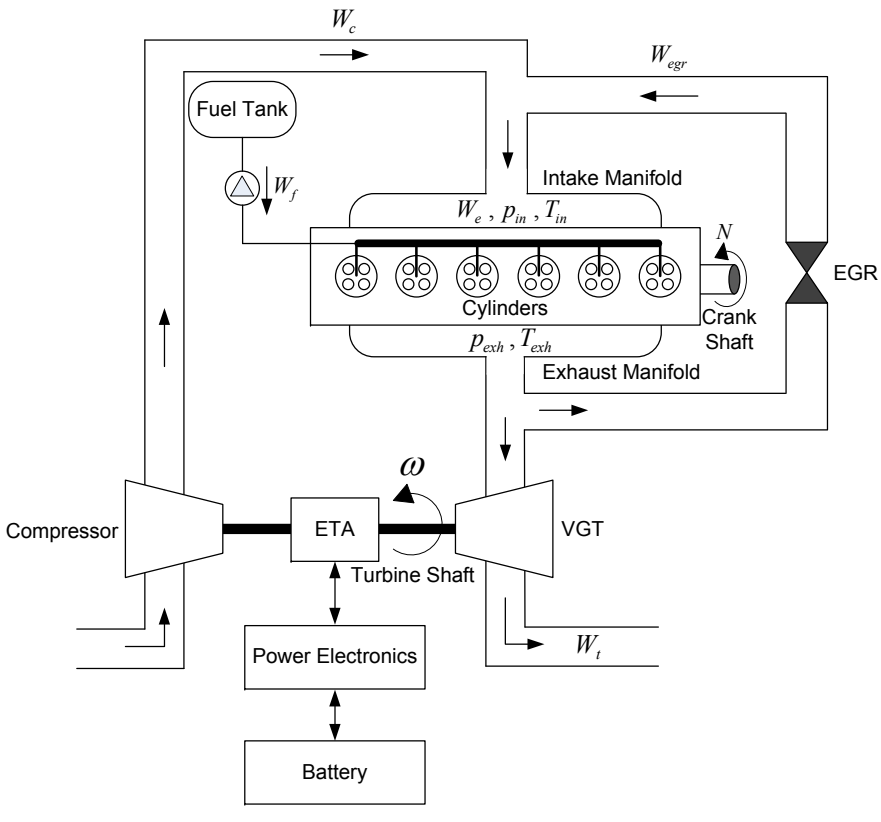

Fig. 1. Electrified turbocharged diesel engine

TABLE I

NOMENCLATURE

\begin{tabular}{lll}
\hline Variable & Description & $\mathrm{Unit}$ \\
\hline$N$ & Engine speed & $\mathrm{rpm}$ \\
$T_{L}$ & Engine load & $\mathrm{Nm}$ \\
$W_{f}$ & Engine fueling rate & $\mathrm{kg} / \mathrm{s}$ \\
$W_{c}$ & Compressor air mass flow rate & $\mathrm{kg} / \mathrm{s}$ \\
$W_{e g r}$ & EGR mass flow rate & $\mathrm{kg} / \mathrm{s}$ \\
$W_{e}$ & Total mass flow rate & $\mathrm{kg} / \mathrm{s}$ \\
$W_{t}$ & Turbine gas mass flow rate & $\mathrm{kg} / \mathrm{s}$ \\
$F_{1}$ & Burnt gas fraction, defined as $\frac{W_{e g r}}{W_{c}+W_{e g r}}$ & - \\
$\lambda$ & In-cylinder air-fuel ratio, defined as $\frac{W_{c}}{W_{f}}$ & - \\
$P_{c}$ & Compressor power & $\mathrm{kW}$ \\
$P_{t}$ & Turbine power & $\mathrm{kW}$ \\
$P_{e m}$ & EM power & $\mathrm{kW}$ \\
$p_{i n}$ & Intake manifold pressure & $\mathrm{kPa}$ \\
$p_{\text {exh }}$ & Exhaust manifold pressure & $\mathrm{kPa}$ \\
$p_{a m}$ & Ambient pressure & $\mathrm{kPa}$ \\
$T_{i n}$ & Intake manifold temperature & $\mathrm{K}$ \\
$T_{e x h}$ & Exhaust manifold temperature & $\mathrm{K}$ \\
$V_{i n}$ & Intake manifold volume & $\mathrm{m} 3$ \\
$V_{e x h}$ & Exhaust manifold volume & $\mathrm{m} 3$ \\
$V_{d}$ & Displacement volume & $\mathrm{m} 3$ \\
$\omega$ & Turbine speed & $\mathrm{rpm}$ \\
$\tau$ & Turbocharger time constant & $\mathrm{s}$ \\
$\eta_{c}$ & Compressor isentropic efficiency & - \\
$\eta_{t}$ & Turbine isentropic efficiency & - \\
$\eta_{m}$ & Turbocharger mechanical efficiency & - \\
$\eta_{v}$ & Volumetric efficiency & - \\
$\chi_{e g r}$ & EGR valve position & - \\
$\chi_{v g t}$ & VGT vane position & - \\
$c_{p}$ & Specific heat at constant pressure, 1.01 & $\mathrm{~kJ} /(\mathrm{kgK})$ \\
$c_{v}$ & Specific heat at constant volume, 0.718 & $\mathrm{~kJ} /(\mathrm{kgK})$ \\
$R_{g}$ & Specific gas constant, $c_{p}-c_{v}$ & $\mathrm{~kJ} /(\mathrm{kgK})$ \\
$\gamma$ & Specific heat ratio, $c_{p} / c_{v}$ & - \\
$\mu$ & ( $\gamma-1) / \gamma$ & - \\
\hline & & \\
\hline
\end{tabular}

steady state boost pressure to enhance low speed torque. In generator mode, the additional turbine torque resulting from exceed exhaust energy causes a power flow to the generator.

The key in air path control is keeping reasonable values

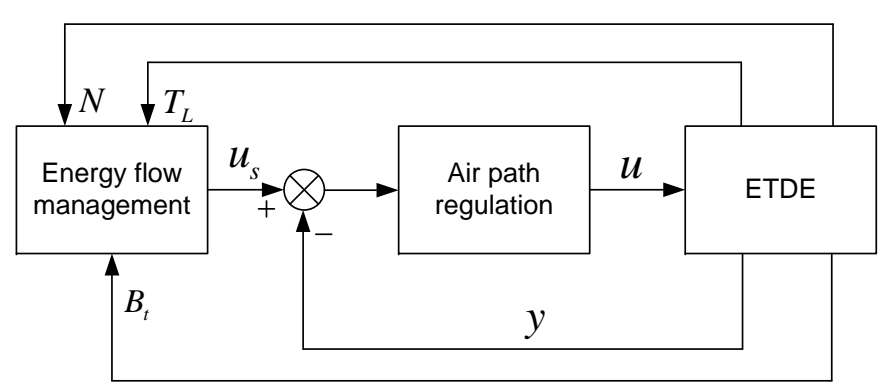

Fig. 2. Control diagram of the ETDE

of $F_{1}$ and $\lambda$ in the intake manifold, to get a good balance between $\mathrm{NO}_{\mathrm{x}}$ and PM. The values of $F_{1}$ and $\lambda$ can be obtained by measuring $W_{c}$ and estimating $W_{e g r}$. Moreover, $p_{\text {exh }}$ is the critical variable in the exhaust manifold. Its value impacts the amount of recoverable exhaust energy and the turbine speed. Therefore, $p_{i n}, W_{c}$, and $p_{\text {exh }}$ are selected as the performance variables in depicting the overall air path dynamics. Ignoring the slow deviation of $T_{i n}$ and $T_{e x h}$, the mean value model of the air path is formulated as:

$$
\begin{aligned}
\dot{p}_{i n} & =\frac{R_{g} T_{i n}}{V_{i n}}\left(W_{c}+W_{e g r}-W_{e}\right), \\
\dot{p}_{e x h} & =\frac{R_{g} T_{e x h}}{V_{e x h}}\left(W_{e}-W_{e g r}-W_{t}+W_{f}\right), \\
\dot{P}_{c} & =\frac{1}{\tau}\left(\eta_{m}\left(P_{t}+P_{e t a}\right)-P_{c}\right) .
\end{aligned}
$$

$W_{e}$ is the total mass flow rate from intake manifold to cylinders and modeled by the speed-density equation as

$$
W_{e}=\frac{\eta_{v} p_{i n} V_{d}}{120 T_{i n} R_{g}} N .
$$

$W_{c}$ is related to $P_{c}$ by

$$
W_{c}=\frac{\eta_{c}}{c_{p} T_{a}} \frac{P_{c}}{\left(\frac{p_{i n}}{p_{a m}}\right)^{\mu}-1},
$$

while $P_{t}$ can be expressed by $W_{t}$ :

$$
P_{t}=\eta_{t} c_{p} T_{e x h}\left(1-\left(\frac{p_{a m}}{p_{\text {exh }}}\right)^{\mu}\right) W_{t} .
$$

The $W_{e g r}$ and $W_{t}$ can be obtained from the orifice equation and turbine mass flow map, respectively, where more details can be found in [12].

\section{PROBLEM FORMULATION}

A systematic solution for the control of an ETDE is based on the optimization of energy flows. Air path variables are tuned in the optimization process in two steps. In the energy flow management step, the optimal setpoints of the air path variables are selected so as to minimize steady state fuel consumption. In the air path regulation step, the variables are regulated so as to track the generated setpoints. The two-step control structure is shown as Fig. 2. 


\section{A. Energy Flow Management}

The primary task of the energy flow management is to formulate the cost function in terms of fuel efficiency. The total fuel power is

$$
P_{a}=H_{\mathrm{LHV}} W_{f},
$$

where $H_{\mathrm{LHV}}$ is the lower heating value of the fuel. Since the energy is generated from both engine cylinders and battery, the instantaneous equivalent consumed power of an ETDE is given by

$$
P_{e q}=P_{a}+s P_{b},
$$

where $P_{b}$ is the battery power, and $s$ is a non-dimensional electricity-fuel energy equivalent factor, which is obtained via off-line calibration in both ETA motoring and generating modes. In the conventional case, the engine brake specific fuel consumption (BSFC) is used as a measure of the engine fuel efficiency:

$$
B_{e}=\frac{W_{f}}{P_{e}}
$$

where $P_{e}$ is the engine power. In an ETDE, the idea of total BSFC is introduced to quantify the fuel efficiency of the complete system including engine and ETA:

$$
B_{t}=B_{e} \frac{P_{e q}}{P_{a}}
$$

Because $B_{t}$ is an instantaneous variable, it can be selected as the cost function

$$
J\left(u_{s}\right)=B_{t},
$$

where $u_{s}$ denotes the control variables set and is selected as

$$
u_{s}=\left[\begin{array}{lll}
\bar{p}_{\text {exh }} & \bar{p}_{i n} & \bar{W}_{c}
\end{array}\right]^{\mathrm{T}},
$$

where $\bar{p}_{e x h}, \bar{p}_{i n}$, and $\bar{W}_{c}$ are the setpoints of $p_{e x h}, p_{i n}$, and $W_{c}$, respectively. The on-line optimization problem is formulated as

$$
\begin{array}{cl}
\min : & J\left(u_{s}\right), \\
\mathrm{s.t}: & u_{s \min } \leq u_{s} \leq u_{s \max },
\end{array}
$$

where $u_{s \min }$ and $u_{s \max }$ denote the permissible limits of the setpoints set. The limits are generated off-line with respect to $N$ and $T_{L}$, under the principle of the legislated exhaust emissions standards are not violated.

\section{B. Air Path Regulation}

The objective of air path regulation is the tracking of the setpoints given in (10). For the highly nonlinear system shown in (1a)-(1c), its piecewise linear models can be identified at different operating regions in the $N-T_{L}$ map. In a specified operating region, the linear model of the ETDE air path can be expressed in the form of discrete state space equations:

$$
\left\{\begin{array}{l}
x(k+1)=A x(k)+B u(k) \\
y(k)=C x(k)
\end{array},\right.
$$

where $x \in \mathbb{R}^{n}$ is the state vector, $u \in \mathbb{R}^{m}$ is the input vector, $y \in \mathbb{R}^{p}$ is the output vector, $(A, B)$ is a controllable pair,

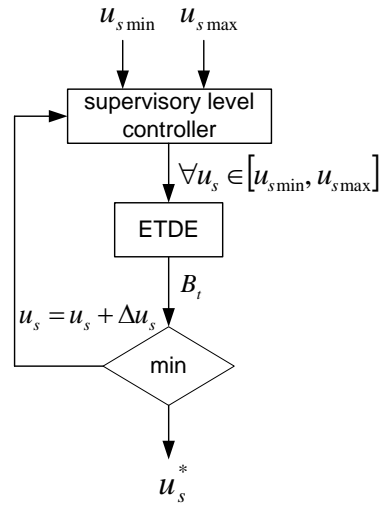

Fig. 3. Workflow of the supervisory level controller

and the coefficient matrices $A, B$, and $C$ are obtained via system identification. The inputs and outputs of the ETDE are selected as

$$
\begin{aligned}
& u=\left[\begin{array}{lll}
P_{\text {eta }} & \chi_{\text {vgt }} & \chi_{\text {egr }}
\end{array}\right]^{\mathrm{T}}, \\
& y=\left[\begin{array}{lll}
p_{\text {exh }} & p_{\text {in }} & W_{c}
\end{array}\right]^{\mathrm{T}},
\end{aligned}
$$

and are bounded as:

$$
\begin{aligned}
& u_{\min } \leq u \leq u_{\max } \\
& y_{\min } \leq y \leq y_{\max }
\end{aligned}
$$

where the limits on $u$ and $y$ are the permissible ranges of actuator position and performance variables. The desired output $\bar{y}$ in (12) is the $u_{s}$ indicated in (10). A closed loop MIMO controller is proposed to function as the air path regulation module of ETDE.

\section{Hierarchical Levels Controllers Design}

Energy flow management and air path regulation are working together as two cascaded modules, where the energy flow management module behaves as the supervisory level controller, and the air path regulation module behaves as a low level controller.

\section{A. Supervisory Level Controller}

The supervisory level controller generates the optimal setpoints $u_{s}^{*}$. However the search process is potentially too lengthy for a real time implementation. A much faster process is proposed consisting of two stages: an off-line static optimization and an on-line search. The static optimization gives a baseline value of $u_{s}$ while the on-line search is restricted to a narrow band around the baseline value.

Off-line calibration is only made on sampled operating points, so the $u_{s}$ value is required to be modified on-line in real-time implementation. The ECMS is utilized to find $u_{s}^{*}$ and its workflow is illustrated in Fig. 3. The tentative values range from $u_{s \min }$ to $u_{s \max }$ with a step $\Delta u_{s}$ :

$$
\Delta u_{s}=\left[\begin{array}{lll}
\Delta \bar{p}_{e x h} & \Delta \bar{p}_{i n} & \Delta \bar{W}_{c}
\end{array}\right]^{\mathrm{T}} .
$$

Each tentative value of $u_{s}$ will be applied on the ETDE with the time period $\Delta t$. The optimal control law obtained will be finally applied to the engine after the search process is 
finished. The values of $u_{s \min }$ and $u_{s \max }$ are determined by imposed constraints on torque, emissions, temperature, and battery state-of-charge (SOC). For each tentative value of $u_{s}$, the cost function $B_{t}$ is computed. The optimal setpoint $u_{s}^{*}$ is selected as the tentative value which yields the minimal value of $B_{t}$.

In the proposed investigation, three variables are to be optimized and there are a lot of possible combinations. This problem can be realized by analyzing the operating principle of an engine. From the physical point of view, $p_{e x h}, p_{i n}$, and $W_{c}$ would change in the same direction. Therefore, the updating step can be set as

$$
\Delta u_{s}=\left[\begin{array}{lll}
K_{1} & K_{2} & 1
\end{array}\right]^{\mathrm{T}} \Delta \bar{W}_{c},
$$

where $K_{1}$ and $K_{2}$ are positive gains and are obtained from fitting the off-line calibration data.

\section{B. Low Level Controller}

EMPC is selected as the low level controller for its capability in dealing with internal couplings and external constraints. To remove the impact of unknown constant disturbance and slowly varying process disturbance on the identified model (12), an augmented model is formulated as

$$
\left\{\begin{array}{l}
{\left[\begin{array}{c}
x(k+1) \\
u(k) \\
u_{s}(k+1)
\end{array}\right]} \\
\underbrace{\left[\begin{array}{ccc}
A & B & 0 \\
0 & I & 0 \\
0 & 0 & I
\end{array}\right]}_{\tilde{x}(k+1)} \underbrace{\left[\begin{array}{c}
x(k) \\
u(k-1) \\
u_{s}(k)
\end{array}\right]}_{\tilde{A}}+\underbrace{\left[\begin{array}{c}
B \\
I \\
0
\end{array}\right]}_{\tilde{x}(k)} \underbrace{\underbrace{\Delta u(k)}_{\tilde{u}}}_{\tilde{B}}, \\
\tilde{y}(k)=\underbrace{\left[\begin{array}{lll}
C & 0 & -I
\end{array}\right]}_{\tilde{C}(k)} \tilde{x}(k),
\end{array}\right.
$$

where $\tilde{x}(k) \in \mathbb{R}^{n+6}, \tilde{u}(k) \in \mathbb{R}^{3}, \tilde{y}(k)=y(k) \in \mathbb{R}^{3}$. For a regulation problem, the system performance index can be specified by a quadratic cost function to be minimized at time instant $k$ :

$$
\begin{aligned}
\min _{\tilde{U}} & J(\tilde{x}(k), \tilde{U})=\underbrace{\left\|\tilde{x}\left(k+H_{p}\right)\right\|_{P}^{2}}_{J\left(\tilde{x}\left(k+H_{p}\right)\right)} \\
& +\sum_{i=0}^{H_{p}-1} \underbrace{\left(\|\tilde{x}(k+i)\|_{Q}^{2}+\|\tilde{u}(k+i)\|_{R}^{2}\right)}_{J(\tilde{x}(k+i), \tilde{u}(k+i))}, \\
\text { s.t. } & \tilde{x}(k+i+1)=\tilde{A} \tilde{x}(k+i)+\tilde{B} \tilde{u}(k+i), \\
& \tilde{u}_{\min } \leq \tilde{u}(k+i) \leq \tilde{u}_{\max }, \quad i=0, \ldots, H_{c}-1, \quad(17 \mathrm{c}) \\
& \tilde{u}(k+i)=\tilde{u}\left(k+H_{c}-1\right), \quad i=H_{c}, \ldots, H_{p}, \quad(17 \mathrm{~d}) \\
& \tilde{y}_{\min } \leq \tilde{y}(k+i) \leq \tilde{y}_{\max }, \quad i=1, \ldots, H_{p},
\end{aligned}
$$

where $\|x\|_{S}^{2}=x^{\mathrm{T}} S x ; H_{p}$ and $H_{c}$ are the prediction horizon and control horizon, respectively; $\tilde{x}(k+i)$ and $\tilde{y}(k+i)$ denote the predictions of $\tilde{x}$ and $\tilde{y}$ at time instant $k+i$, made at time instant $k$, respectively; $\tilde{u}(k+i)$ is the value of control input $\tilde{u}$ at time instant $k+i ; \tilde{U}=\left[\tilde{u}^{\mathrm{T}}(k), \ldots, \tilde{u}^{\mathrm{T}}(k+\right.$ $\left.\left.H_{p}-1\right)\right]^{\mathrm{T}} \in \mathbb{R}^{3 H_{p}}$ is the control sequence within which the optimal control inputs be determined; $\tilde{X}=\left[\tilde{x}^{\mathrm{T}}(k+\right.$ $\left.1), \ldots, \tilde{x}^{\mathrm{T}}\left(k+H_{p}\right)\right]^{\mathrm{T}} \in \mathbb{R}^{(n+6) H_{p}}$ is the vector of predicted states; $J\left(\tilde{x}\left(k+H_{p}\right)\right.$ is the terminal penalty function, while $J(\tilde{x}(k+i), \tilde{u}(k+i))$ is the stage cost at time instant $k+i$; From physical point of view, $H_{c} \leq H_{p}$ should be guaranteed. $P=P^{\mathrm{T}}>0, Q=Q^{\mathrm{T}}>0$ and $R=R^{\mathrm{T}}>0$ are also assumed.

The cost function (17a) can be re-written in the following quadratic form:

$$
J(\tilde{x}(k), \tilde{U})=\tilde{X} \bar{Q} \tilde{X}+\tilde{U} \bar{R} \tilde{U},
$$

where $\bar{Q}=\operatorname{diag}(Q, \ldots, Q, P)$ and $\bar{R}=\operatorname{diag}(R, \ldots, R)$. Using linear transformation, the optimization problem (18) can be converted into a quadratic programming (QP) problem. Employing the multi-parametric technique, the complex plane of $\tilde{X}$ can be divided into multiple convex polygons. Applying the Karush-Kuhn-Tucker (KKT) optimality conditions, the optimal control law $\tilde{U}^{*}(k)$ is obtained explicitly as affine functions of $\tilde{x}(k)$ from the generated polygons. For a tracking problem, the cost fuction (17a) can be re-formulated as

$$
J(\tilde{y}(k), \tilde{U})=\sum_{i=0}^{H_{p}-1}\left(\|\tilde{y}(k+i)-\bar{y}\|_{Q}^{2}+\|\tilde{u}(k+i)\|_{R}^{2}\right),
$$

while the other constraints in (17) are kept the same.

The multi-parametric QP (mpQP) problem is solved offline and the control laws are stored in look-up tables. In the on-line implementation stage, control laws are read from the table without conducting any optimization. This grants the EMPC an advantage of reduced computation time meanwhile maintaining the identical performance with MPC. In real time control, only the first component of the vector $\tilde{U}^{*}(k)$ would be applied:

$$
\tilde{u}(k)=\left[\begin{array}{llll}
I & 0 & \ldots & 0
\end{array}\right] \tilde{U}^{*}(k) .
$$

In the next step, $\tilde{U}^{*}(k+1)$ is called from the look-up table again, and $\tilde{u}(k+1)$ is updated accordingly. To estimate the states $\tilde{x}(k)$, a standard Kalman filter is employed.

\section{VAlidation Results}

Simulations have been carried out on a physical plant model built in Dynasty, a proprietary multi physics simulation software package used within Caterpillar. The engine manifolds are modeled as one-dimensional components, permitting to capture the pulsations caused by engine actuators operation. The compressor, turbine, and ETA are all represented as map based models. The controllers are built in Matlab/Simulink. The investigated engine is a six-cylinder, 7.1-L heavy duty engine with rated power of $225 \mathrm{~kW}$ at $2200 \mathrm{rpm}$ and a rated torque of $1280 \mathrm{Nm}$ at $1400 \mathrm{rpm}$.

In the evaluation of the tracking performance of the low level controller, the comparisons of the proposed EMPC method and the distributed PID control method are tested at $1600 \mathrm{rpm}, 500 \mathrm{Nm}$, where the EMPC law is computed by an open-source MPT toolbox [13]. In the test of the systematic solution, the engine operating point switches between $1600 \mathrm{rpm}, 500 \mathrm{Nm}$ and $1760 \mathrm{rpm}, 404 \mathrm{Nm}$. The tested points locate in the main operating range of the ETDE. 


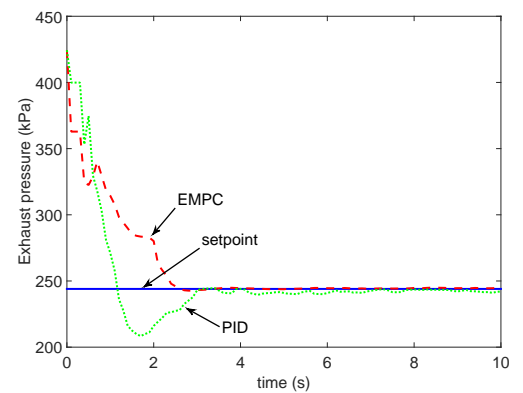

(a) $p_{\text {exh }}$

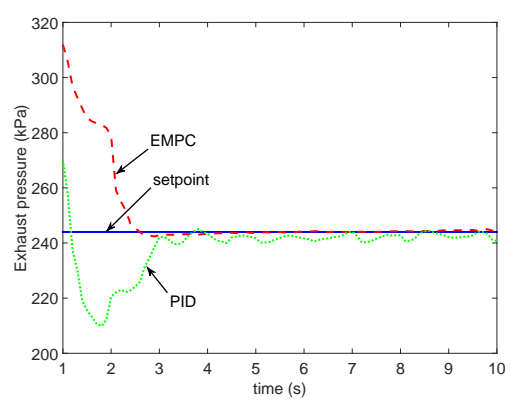

(a) $p_{\text {exh }}$

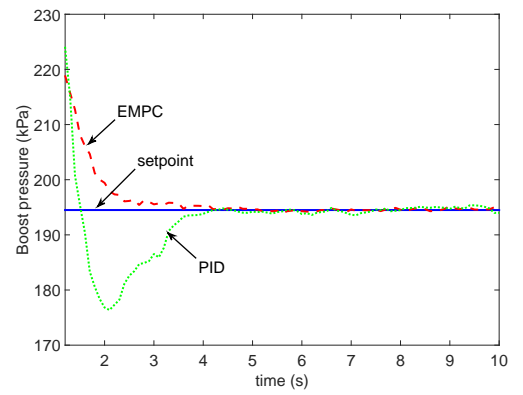

(b) $p_{\text {in }}$

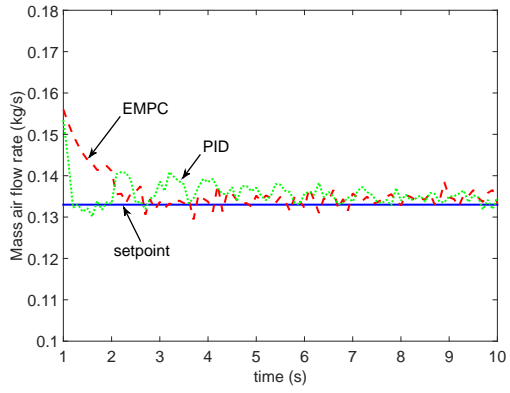

(c) $W_{c}$

Fig. 4. Low level control results

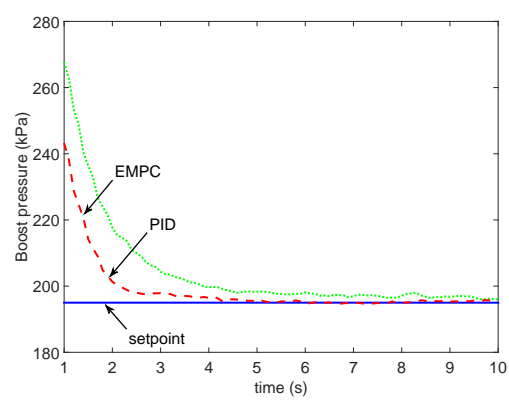

(b) $p_{\text {in }}$

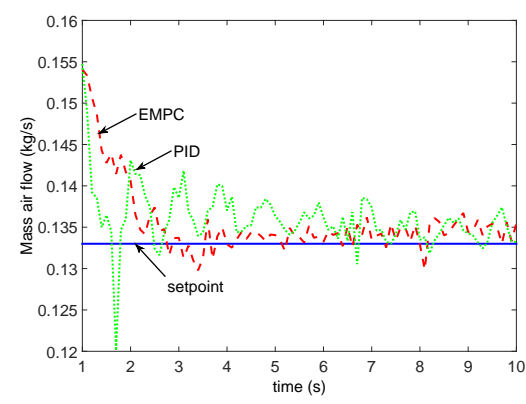

(c) $W_{c}$

Fig. 5. Tracking performance comparison with constraint on $\chi_{v g t}$

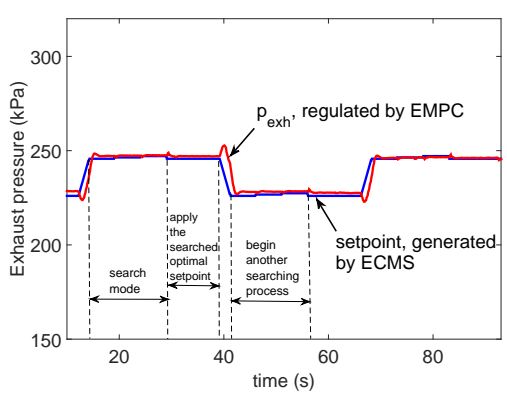

(a) $p_{\text {exh }}$

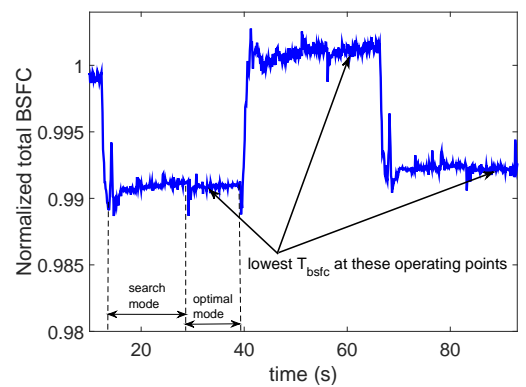

(b) $B_{t}$

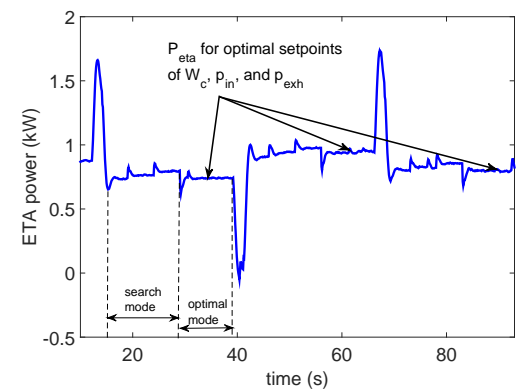

(c) Peta

Fig. 6. Hierarchical levels control results

\section{A. Performance Evaluation of Low Level Controller}

In the evaluation of the low level controller, a $3 \mathrm{I} 3 \mathrm{O}$ PID control method is employed as the baseline, by which $p_{e x h}, p_{i n}$, and $W_{c}$ are controlled by $P_{\text {eta }}, \chi_{v g t}$, and $\chi_{e g r}$, respectively. The constraints on the control signals $u$ are set as

$$
\begin{aligned}
u_{\min } & =\left[\begin{array}{lll}
-2 \mathrm{~kW} & 0 & 0
\end{array}\right]^{\mathrm{T}}, \\
u_{\max } & =\left[\begin{array}{lll}
2 \mathrm{~kW} & 95 \% & 100 \%
\end{array}\right]^{\mathrm{T}} .
\end{aligned}
$$

The setpoints to be tracked are taken from existing engine calibration data with the values of

$$
\bar{y}=\left[\begin{array}{lll}
244 \mathrm{kPa} & 195 \mathrm{kPa} & 0.133 \mathrm{~kg} / \mathrm{s}
\end{array}\right]^{\mathrm{T}} .
$$

The prediction horizon and control horizon in the EMPC controller are set as $H_{p}=5$ and $H_{c}=2$ respectively. The gain matrices in (19) are set as $Q=0.4 I_{3}$ and
$R=0.5 I_{3}$, respectively. In PID controllers, the gain parameters are tuned by experience based the baseline of Ziegler-Nichols method. The couplings among multiple PID controllers increase the difficulty in tuning gain values. The control results from both methods are shown as Fig. 4. The deviations at the start period are because of the initialization of the engine. It can be observed that with all the 3 target variables, the tracking performance of EMPC is smoother and faster. The controlled variables converge to the steady values asymptotically reaching the setpoints in around $2.8 \mathrm{~s}$. In contrast, the overshoots are high and the settling time is longer with the PID method, due to the PID gain values being set relatively high to reduce convergence time. In the EMPC method, the control action is the pre-computed optimal control law, such that the system trajectory represents the best achievable balance of regulation error and control 
effort.

\section{B. Performance Evaluation with Input Constraints}

The capability of EMPC in dealing with actuator saturation constraints is demonstrated in Fig. 5, where the VGT vane opening is constrained to $\chi_{v g t} \in[0,32 \%]$. It can be observed that with the PID method, the tracking performance deteriorates for all the three variables. In Fig. 5(b), the steady state error of using PID method cannot be diminished in a reasonable time while $p_{i n}$ is directly controlled by $\chi_{v g t}$. The VGT vane cannot open sufficiently to reduce $p_{i n}$ to the desired value. Moreover, in Fig. 5(c) and Fig. 5(a), the oscillations and steady state errors are both greater for the PID implementation. As a comparison, using the EMPC method, the performance is much less affected, due to the actions of the other two actuators would be tuned considering the constraint on $\chi_{v g t}$.

\section{Performance Evaluation of Systematic Solution}

In the evaluation of the proposed systematic solution, the ETDE is tested under two different operating conditions. For these tests, the setpoints are restricted within the band of

$$
\bar{u}_{s}-\Delta u_{s} \leq u_{s} \leq \bar{u}_{s}+\Delta u_{s}
$$

where $\bar{u}_{s}$ is the setpoint generated by the off-line calibration data. The steps of the updated setpoints are set as

$$
\Delta u_{s}=\left[\begin{array}{lll}
0.7 \mathrm{kPa} & 0.5 \mathrm{kPa} & 0.001 \mathrm{~kg} / \mathrm{s}
\end{array}\right]^{\mathrm{T}} .
$$

Each tentative value of $u_{s}$ is applied to the ETDE with the period of $\Delta t=5 \mathrm{~s}$. In Fig. 6(a), the tuning and tracking process of $p_{\text {exh }}$ is selected. In the search mode, $u_{s}$ is updated according to the specified pattern. The ECMS computes the corresponding $B_{t}$. Meanwhile, the actual $p_{\text {exh }}$ on engine is regulated by the low level EMPC controller to track the given $u_{s}$. EMPC is fast enough to follow the change of $u_{s}$. When the search process ends, the calculated optimal setpoint $u_{s}^{*}$ with lowest $B_{t}$ is applied to the ETDE until the operating condition changes. When the ECMS module detects that the ETDE has a new operating condition, a new searching process is started.

The changes on $B_{t}$ are illustrated in Fig. 6(b). The $B_{t}$ in the optimal mode is identical to the lowest $B_{t}$ in the search mode. The actuator action is given by the EMPC controller, where the tuning process of the ETA power is illustrated in Fig. 6(c). $P_{\text {eta }}$ is an electrical variable and therefore has a very low inertia. It can be seen that $P_{\text {eta }}$ achieves its steady value very quickly.

\section{CONCLUSION}

A systematic solution for energy flow distribution and the air path regulation of a newly designed ETDE is proposed in this paper. The optimal energy flow distribution is completed in a cascaded static stage and a real-time tuning stage. The EMPC behaves as a MIMO low level controller considering practical constraints. The simulation of a physical model via a cross-platform simulation technique validated the effectiveness of the proposed method. Developing a global nonlinear control method based on explicit modeling to replace the piecewise linear controller as the low level controller is the proposed future direction.

\section{ACKNOWLEDGEMENT}

This work was co-funded by the Technology Strategy Board (TSB) UK, under a grant for the Low Carbon Vehicle IDP4 Programme (TP14/LCV/6/I/BG011L). The Technology Strategy Board is an executive body established by the United Kingdom Government to drive innovation. It promotes and invests in research, development and the exploitation of science, technology and new ideas for the benefit of business - increasing sustainable economic growth in the UK and improving quality of life.

Thanks also go to our consortium partners and subcontractors.

\section{REFERENCES}

[1] S. Bilgen, "Structure and environmental impact of global energy consumption," Renewable and Sustainable Energy Reviews, vol. 38, pp. 890-902, 2014.

[2] R. Stobart and R. Weerasinghe, "Heat recovery and bottoming cycles for SI and CI engines - a perspective," SAE International, pp. 200601-0662, 2006.

[3] U. Hopmann and M. Algrain, "Diesel engine electric turbo compound technology," SAE International, pp. 2003-01-2294, 2003.

[4] S. Arnold, C. Balis, P. Barthelet, E. Poix, T. Samad, G. Hampson, and S. Shahed, "Garrett electric boosting systems (EBS) program," Honeywell Turbo Technologies, Rolle, Switzerland, Tech. Rep. DEFC05-00OR22809, June 2005.

[5] S. Ibaraki, Y. Yamashita, K. Sumida, H. Ogita, and Y. Jinnai, "Development of the hybrid turbo, an electrically assisted turbocharger," Mitsubishi Heavy Industries Technical Review, vol. 43, no. 3, pp. 1-5, 2006.

[6] I. Kolmanovsky, A. Stefanopoulous, and B. Powell, "Improving turbocharged diesel engine operation with turbo power assist system," in Proceedings of the IEEE International Conference on Control Applications, 1999, pp. 454-459.

[7] J. Panting, K. Pullen, and R. Martinez-Botas, "Turbocharged motorgenerator for improvement of transient performance in an internal combustion engine," Proceedings of the Institution of Mechanical Engineers Part D-Journal of Automobile Engineering, vol. 215, no. 3, pp. 369-383, 2001.

[8] F. Millo, F. Mallamo, and E. Pautasso, "The potential of electric exhaust gas turbocharging for HD diesel engines," SAE International, pp. 2006-01-0437, 2006.

[9] D. Hountalas, C. Katsanos, and V. Lamaris, "Recovering energy from the diesel engine exhaust using mechanical and electrical turbocompounding," SAE International, pp. 2007-01-1563, 2007.

[10] N. Terdich and R. Martinez-Botas, "Experimental efficiency characterization of an electrically assisted turbocharger," SAE International, pp. 2013-24-0122, 2013

[11] A. Costall, R. Ivanov, and T. Langley, "Electric turbo assist: efficient rapid boost for heavy duty diesel engines," in Proceedings of the Conference on Thermo and Fluid Dynamic Processes in Direct Injection Engines, 2012, pp. 1-18.

[12] M. Jankovic and I. Kolmanovsky, "Constructive Lyapunov control design for turbocharged diesel engines," IEEE Transactions on Control Systems Technology, vol. 8, no. 2, pp. 288-299, 2000.

[13] (2014, July) Multi-parametric toolbox 3. [Online]. Available: http://people.ee.ethz.ch/ mpt/3/ 